\title{
Supporting Information for: "Using Atomic Confining Potentials for Geometry Optimization and Vibrational Frequency Calculations in Quantum-Chemical Models of Enzyme Active Sites"
}

\author{
Saswata Dasgupta and John M. Herbert* \\ Department of Chemistry and Biochemistry, The Ohio State University, Columbus, Ohio \\ 43210 USA
}

January 20, 2020

*herbert@chemistry.ohio-state.edu 
Table S1: Comparison vibrational frequencies for Gly-Ala. with both terminal carbons confined with potential, computed using analytic and finite-difference second derivatives.

\begin{tabular}{|c|c|c|c|c|c|c|c|c|c|c|c|c|c|c|c|}
\hline \multirow{3}{*}{ mode } & \multicolumn{5}{|c|}{$k=30 \mathrm{~N} / \mathrm{m}$} & \multicolumn{5}{|c|}{$\bar{c} k=450 \mathrm{~N} / \mathrm{m}$} & \multicolumn{5}{|c|}{$\overline{c k}=990 \mathrm{~N} / \mathrm{m}$} \\
\hline & \multicolumn{3}{|c|}{ freq. $\left(\mathrm{cm}^{-1}\right)$} & \multicolumn{2}{|c|}{ error $\left(\mathrm{cm}^{-1}\right.$} & \multicolumn{3}{|c|}{ freq. $\left(\mathrm{cm}^{-1}\right)$} & \multicolumn{2}{|c|}{ error $\left(\mathrm{cm}^{-1}\right.$} & \multicolumn{3}{|c|}{ freq. $\left(\mathrm{cm}^{-1}\right)$} & \multicolumn{2}{|c|}{ error $\left(\mathrm{cm}^{-1}\right.$} \\
\hline & analytic & IDRV $=0^{a}$ & $\mathrm{IDRV}=1^{b}$ & IDRV $=0^{a}$ & IDRV $=1^{b}$ & analytic & $\mathrm{IDRV}=0^{a}$ & IDRV $=1^{b}$ & IDRV $=0^{a}$ & IDRV $=1^{b}$ & analytic & IDRV $=0^{a}$ & IDRV $=1^{b}$ & IDRV $=0^{a}$ & IDRV $=1^{b}$ \\
\hline 1 & 70.12 & 70.12 & 70.08 & 0.00 & 0.04 & 90.49 & 90.48 & 90.52 & -0.04 & -0.03 & 84.23 & 84.12 & 84.34 & 0.11 & -0.11 \\
\hline 2 & 88.21 & 88.19 & 88.21 & 0.02 & 0.00 & 137.97 & 137.96 & 138.00 & 0.02 & -0.03 & 95.47 & 95.47 & 95.48 & 0.00 & -0.01 \\
\hline 3 & 141.08 & 141.07 & 141.10 & 0.01 & -0.02 & 182.44 & 182.40 & 182.49 & 0.03 & -0.05 & 167.33 & 167.31 & 167.35 & 0.02 & -0.02 \\
\hline 4 & 264.43 & 264.42 & 264.46 & 0.01 & -0.03 & 264.31 & 264.32 & 264.30 & 0.04 & 0.01 & 267.91 & 267.91 & 267.92 & 0.00 & -0.01 \\
\hline 5 & 285.17 & 285.16 & 285.17 & 0.01 & 0.00 & 311.79 & 311.80 & 311.83 & 0.01 & -0.04 & 326.65 & 326.64 & 326.68 & 0.01 & -0.03 \\
\hline 6 & 291.15 & 291.11 & 291.15 & 0.04 & 0.00 & 332.59 & 332.59 & 332.61 & 0.04 & -0.02 & 351.48 & 351.48 & 351.51 & 0.00 & -0.03 \\
\hline 7 & 326.40 & 326.40 & 326.40 & 0.00 & 0.00 & 395.34 & 395.35 & 395.35 & 0.00 & -0.01 & 407.03 & 407.03 & 407.02 & 0.00 & 0.01 \\
\hline 8 & 349.19 & 349.18 & 349.18 & 0.01 & 0.01 & 434.71 & 434.71 & 434.73 & 0.00 & -0.02 & 492.38 & 492.37 & 492.38 & 0.01 & 0.00 \\
\hline 9 & 413.53 & 413.53 & 413.55 & 0.00 & -0.02 & 486.11 & 486.10 & 486.11 & 0.02 & 0.00 & 546.13 & 546.13 & 546.16 & 0.00 & -0.03 \\
\hline 10 & 473.06 & 473.05 & 473.08 & 0.01 & -0.02 & 559.27 & 559.27 & 559.29 & 0.03 & -0.02 & 617.96 & 617.96 & 617.99 & 0.00 & -0.03 \\
\hline 11 & 504.75 & 504.75 & 504.76 & 0.00 & -0.01 & 574.93 & 574.93 & 574.93 & 0.01 & 0.00 & 659.66 & 659.66 & 659.69 & 0.00 & -0.03 \\
\hline 12 & 658.69 & 658.68 & 658.73 & 0.01 & -0.04 & 658.18 & 658.18 & 658.20 & 0.05 & -0.02 & 680.86 & 680.86 & 680.86 & 0.00 & 0.00 \\
\hline 13 & 680.82 & 680.82 & 680.82 & 0.00 & 0.00 & 680.68 & 680.68 & 680.67 & 0.00 & 0.01 & 691.78 & 691.78 & 691.78 & 0.00 & 0.00 \\
\hline 14 & 753.20 & 753.20 & 753.24 & 0.00 & -0.04 & 754.44 & 754.44 & 754.44 & 0.04 & 0.00 & 762.07 & 762.07 & 762.06 & 0.00 & 0.01 \\
\hline 15 & 790.84 & 790.83 & 790.84 & 0.01 & 0.00 & 790.30 & 790.29 & 790.31 & 0.01 & -0.01 & 790.33 & 790.32 & 790.33 & 0.01 & 0.00 \\
\hline 16 & 833.35 & 833.35 & 833.33 & 0.00 & 0.02 & 826.02 & 826.01 & 826.04 & -0.02 & -0.02 & 816.47 & 816.46 & 816.51 & 0.01 & -0.04 \\
\hline 17 & 851.34 & 851.33 & 851.32 & 0.01 & 0.02 & 840.19 & 840.18 & 840.19 & -0.01 & 0.00 & 841.21 & 841.20 & 841.21 & 0.01 & 0.00 \\
\hline 18 & 887.46 & 887.46 & 887.50 & 0.00 & -0.04 & 892.17 & 892.17 & 892.20 & 0.04 & -0.03 & 897.35 & 897.34 & 897.39 & 0.01 & -0.04 \\
\hline 19 & 905.80 & 905.79 & 905.82 & 0.01 & -0.02 & 931.92 & 931.92 & 931.88 & 0.03 & 0.04 & 932.56 & 932.56 & 932.53 & 0.00 & 0.03 \\
\hline 20 & 931.93 & 931.93 & 931.92 & 0.00 & 0.01 & 960.35 & 960.35 & 960.37 & -0.01 & -0.02 & 984.67 & 984.67 & 984.68 & 0.00 & -0.01 \\
\hline 21 & 959.01 & 959.01 & 959.00 & 0.00 & 0.01 & 1021.35 & 1021.35 & 1021.31 & -0.01 & 0.04 & 1023.13 & 1023.13 & 1023.10 & 0.00 & 0.03 \\
\hline 22 & 1020.34 & 1020.34 & 1020.40 & 0.00 & -0.06 & 1045.70 & 1045.71 & 1045.70 & 0.06 & 0.00 & 1088.19 & 1088.19 & 1088.14 & 0.00 & 0.05 \\
\hline 23 & 1032.95 & 1032.95 & 1032.99 & 0.00 & -0.04 & 1074.23 & 1074.23 & 1074.23 & 0.04 & 0.00 & 1115.60 & 1115.60 & 1115.63 & 0.00 & -0.03 \\
\hline 24 & 1108.54 & 1108.54 & 1108.52 & 0.00 & 0.02 & 1121.64 & 1121.64 & 1121.67 & -0.02 & -0.03 & 1125.98 & 1125.98 & 1126.04 & 0.00 & -0.06 \\
\hline 25 & 1122.24 & 1122.24 & 1122.26 & 0.00 & -0.02 & 1143.15 & 1143.15 & 1143.11 & 0.02 & 0.04 & 1151.04 & 1151.04 & 1151.12 & 0.00 & -0.08 \\
\hline 26 & 1132.38 & 1132.38 & 1132.37 & 0.00 & 0.01 & 1152.16 & 1152.16 & 1152.22 & -0.01 & -0.06 & 1184.79 & 1184.78 & 1184.85 & 0.01 & -0.06 \\
\hline 27 & 1152.66 & 1152.66 & 1152.69 & 0.00 & -0.03 & 1160.19 & 1160.19 & 1160.22 & 0.03 & -0.03 & 1209.90 & 1209.90 & 1209.89 & 0.00 & 0.01 \\
\hline 28 & 1217.70 & 1217.70 & 1217.73 & 0.00 & -0.03 & 1258.43 & 1258.43 & 1258.42 & 0.03 & 0.01 & 1272.35 & 1272.34 & 1272.33 & 0.01 & 0.02 \\
\hline 29 & 1258.59 & 1258.60 & 1258.59 & -0.01 & 0.00 & 1270.24 & 1270.24 & 1270.27 & -0.01 & -0.03 & 1284.52 & 1284.52 & 1284.55 & 0.00 & -0.03 \\
\hline 30 & 1276.66 & 1276.66 & 1276.64 & 0.00 & 0.02 & 1282.67 & 1282.67 & 1282.69 & -0.02 & -0.02 & 1297.55 & 1297.55 & 1297.48 & 0.00 & 0.07 \\
\hline 31 & 1293.51 & 1293.51 & 1293.58 & 0.00 & -0.07 & 1304.88 & 1304.88 & 1304.83 & 0.07 & 0.05 & 1337.10 & 1337.09 & 1337.04 & 0.01 & 0.06 \\
\hline 32 & 1342.99 & 1342.98 & 1342.85 & 0.01 & 0.14 & 1343.33 & 1343.32 & 1343.23 & -0.13 & 0.10 & 1376.48 & 1376.48 & 1376.44 & 0.00 & 0.04 \\
\hline 33 & 1385.21 & 1385.21 & 1385.35 & 0.00 & -0.14 & 1391.94 & 1391.94 & 1391.98 & 0.14 & -0.04 & 1394.16 & 1394.16 & 1394.19 & 0.00 & -0.03 \\
\hline 34 & 1397.29 & 1397.28 & 1397.19 & 0.01 & 0.10 & 1409.73 & 1409.73 & 1409.75 & -0.09 & -0.02 & 1417.42 & 1417.42 & 1417.46 & 0.00 & -0.04 \\
\hline 35 & 1419.28 & 1419.28 & 1419.30 & 0.00 & -0.02 & 1424.09 & 1424.08 & 1424.13 & 0.02 & -0.04 & 1438.17 & 1438.16 & 1438.20 & 0.01 & -0.03 \\
\hline 36 & 1443.26 & 1443.26 & 1443.24 & 0.00 & 0.02 & 1445.70 & 1445.70 & 1445.72 & -0.02 & -0.02 & 1448.61 & 1448.61 & 1448.64 & 0.00 & -0.03 \\
\hline 37 & 1448.19 & 1448.19 & 1448.23 & 0.00 & -0.04 & 1503.31 & 1503.30 & 1503.30 & 0.04 & 0.01 & 1505.01 & 1505.00 & 1505.00 & 0.01 & 0.01 \\
\hline 38 & 1505.89 & 1505.89 & 1505.89 & 0.00 & 0.00 & 1525.03 & 1525.03 & 1525.06 & 0.00 & -0.03 & 1525.72 & 1525.72 & 1525.74 & 0.00 & -0.02 \\
\hline 39 & 1518.97 & 1518.98 & 1518.98 & -0.01 & -0.01 & 1532.25 & 1532.25 & 1532.24 & 0.00 & 0.01 & 1552.08 & 1552.07 & 1552.08 & 0.01 & 0.00 \\
\hline 40 & 1524.55 & 1524.54 & 1524.57 & 0.01 & -0.02 & 1548.60 & 1548.60 & 1548.62 & 0.03 & -0.02 & 1575.16 & 1575.16 & 1575.16 & 0.00 & 0.00 \\
\hline 41 & 1531.23 & 1531.22 & 1531.23 & 0.01 & 0.00 & 1552.34 & 1552.34 & 1552.34 & 0.01 & 0.00 & 1628.56 & 1628.56 & 1628.52 & 0.00 & 0.04 \\
\hline 42 & 1620.92 & 1620.92 & 1620.84 & 0.00 & 0.08 & 1627.09 & 1627.09 & 1627.08 & -0.08 & 0.01 & 1672.22 & 1672.21 & 1672.23 & 0.01 & -0.01 \\
\hline 43 & 1699.22 & 1699.21 & 1699.23 & 0.01 & -0.01 & 1698.92 & 1698.92 & 1698.95 & 0.02 & -0.03 & 1703.64 & 1703.63 & 1703.65 & 0.01 & -0.01 \\
\hline 44 & 3017.93 & 3017.93 & 3017.95 & 0.00 & -0.02 & 3023.10 & 3023.12 & 3023.12 & 0.02 & -0.02 & 3019.32 & 3019.32 & 3019.34 & 0.00 & -0.02 \\
\hline 45 & 3033.95 & 3033.96 & 3033.97 & -0.01 & -0.02 & 3028.49 & 3028.50 & 3028.53 & 0.01 & -0.04 & 3027.06 & 3027.05 & 3027.10 & 0.01 & -0.04 \\
\hline 46 & 3043.17 & 3043.18 & 3043.20 & -0.01 & -0.03 & 3035.23 & 3035.23 & 3035.24 & 0.02 & -0.01 & 3037.55 & 3037.55 & 3037.56 & 0.00 & -0.01 \\
\hline 47 & 3077.55 & 3077.55 & 3077.53 & 0.00 & 0.02 & 3076.83 & 3076.84 & 3076.83 & -0.02 & 0.00 & 3075.11 & 3075.11 & 3075.11 & 0.00 & 0.00 \\
\hline 48 & 3103.11 & 3103.12 & 3103.11 & -0.01 & 0.00 & 3083.44 & 3083.45 & 3083.41 & -0.01 & 0.03 & 3084.11 & 3084.11 & 3084.07 & 0.00 & 0.04 \\
\hline 49 & 3135.88 & 3135.89 & 3135.84 & -0.01 & 0.04 & 3112.71 & 3112.72 & 3112.69 & -0.05 & 0.02 & 3121.86 & 3121.87 & 3121.85 & -0.01 & 0.01 \\
\hline 50 & 3256.18 & 3256.18 & 3256.12 & 0.00 & 0.06 & 3251.43 & 3251.43 & 3251.44 & -0.06 & -0.01 & 3250.47 & 3250.47 & 3250.52 & 0.00 & -0.05 \\
\hline 51 & 3261.20 & 3261.21 & 3261.15 & -0.01 & 0.05 & 3269.27 & 3269.28 & 3269.23 & -0.06 & 0.04 & 3271.25 & 3271.26 & 3271.21 & -0.01 & 0.04 \\
\hline 52 & 3447.43 & 3447.44 & 3447.45 & -0.01 & -0.02 & 3470.88 & 3470.88 & 3470.89 & 0.01 & -0.01 & 3476.45 & 3476.46 & 3476.47 & -0.01 & -0.02 \\
\hline 53 & 3536.94 & 3536.94 & 3536.93 & 0.00 & 0.01 & 3574.04 & 3574.04 & 3574.02 & -0.01 & 0.02 & 3581.63 & 3581.63 & 3581.61 & 0.00 & 0.02 \\
\hline 54 & 3636.12 & 3636.14 & 3636.12 & -0.02 & 0.00 & 3640.85 & 3640.86 & 3640.84 & -0.02 & 0.01 & 3640.07 & 3640.08 & 3640.06 & -0.01 & 0.01 \\
\hline
\end{tabular}

Table S2: Anchor atoms indices for all the model systems. The anchor atoms coordinates either kept fixed (coordinate lock formalism) or constrained with a harmonic confiner

\begin{tabular}{c|ccccccccccccccccc}
\hline \hline Model & \multicolumn{11}{c}{ Anchor atom indices } \\
\hline AMDase (Model I) & 3 & 9 & 17 & 20 & 23 & & & & & & & & & \\
AMDase (Model II) & 3 & 14 & 18 & 19 & 22 & 33 & 37 & 44 & 50 & 51 & 55 & 62 & 69 & 70 & 71 & 74 & 84 \\
4-OT & 17 & 18 & 22 & 27 & 28 & 34 & & & & & & & & & & & \\
\hline
\end{tabular}




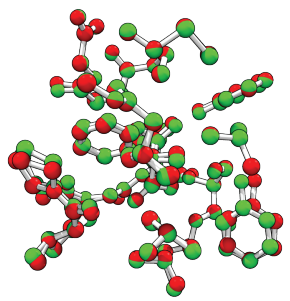

$R$ reactant

$\mathrm{RMSD}=0.27 \AA$

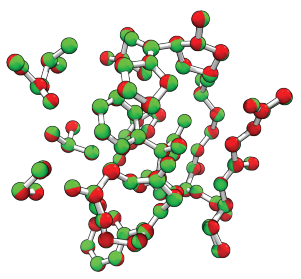

$S$ reactant

$\mathrm{RMSD}=0.06 \AA$

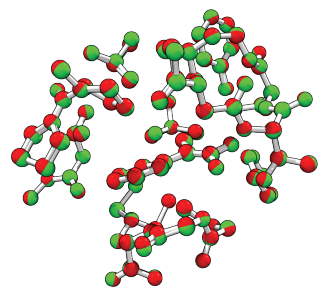

$R$ intermediate

$\mathrm{RMSD}=0.35 \AA$

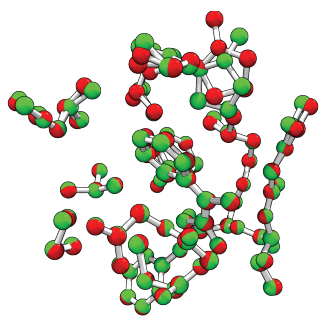

$S$ intermediate

$\mathrm{RMSD}=0.33 \AA$

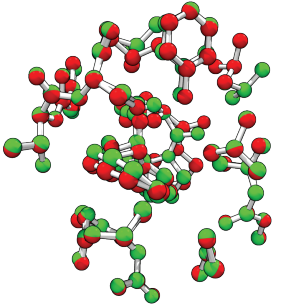

$R$ product

$\mathrm{RMSD}=0.32 \AA$

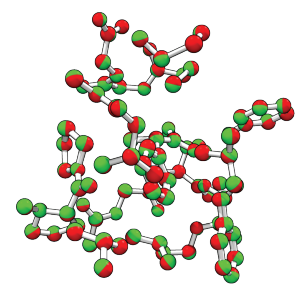

$S$ product

$\mathrm{RMSD}=0.07 \AA$

Figure S1: Optimized structures for model II of methyl(phenyl)malonate in the active site of AMDase, overlaying the structures obtained using harmonic and fixed-atom constraints.
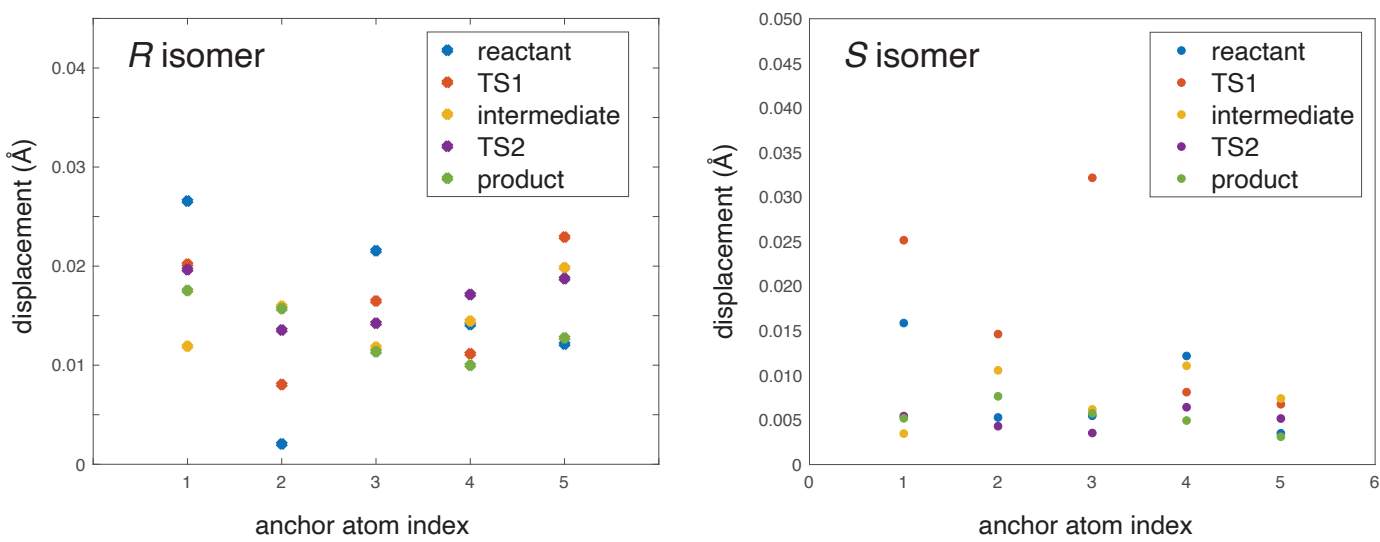

Figure S2: Displacements of the constrained "anchor atoms" as compared to their initial, crystallographic positions $\mathbf{r}_{i}^{0}$, for model I of methyl(phenyl)malonate in AMDase. 

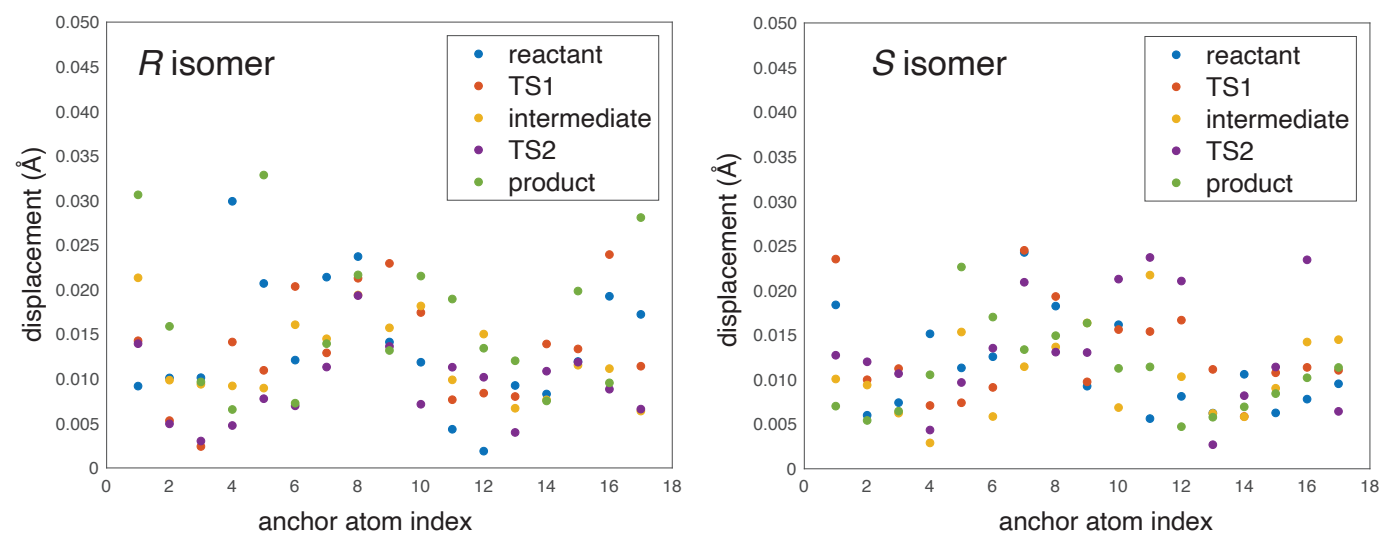

Figure S3: Displacements of the constrained "anchor atoms" as compared to their initial, crystallographic positions $\mathbf{r}_{i}^{0}$, for model II of methyl(phenyl)malonate in AMDase.

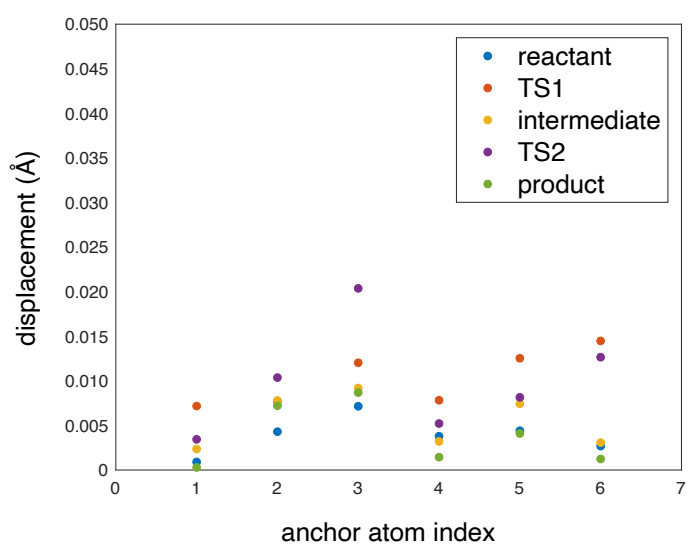

Figure S4: Displacements of the constrained "anchor atoms" as compared to their initial, crystallographic positions $\mathbf{r}_{i}^{0}$, for the reaction catalyzed by 4 -OT. 

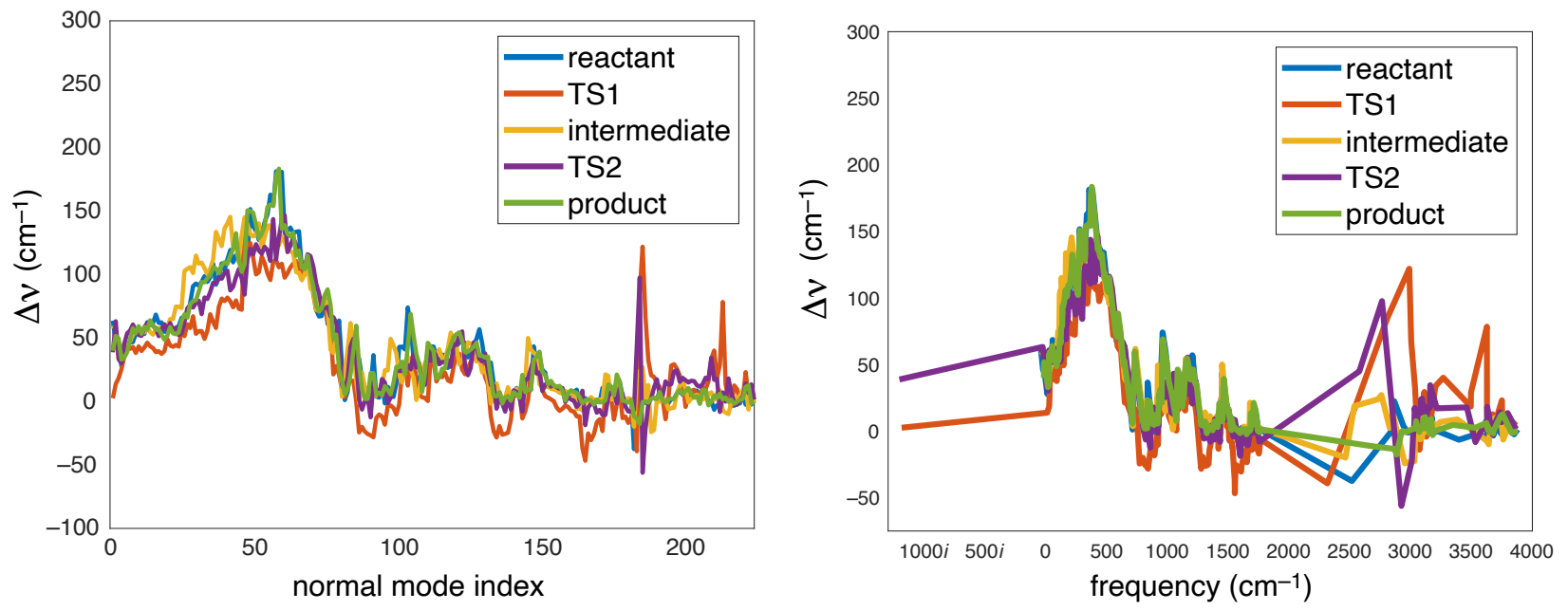

Figure S5: Differences in vibrational frequencies, $\Delta \nu=\nu$ (harmonic conf.) $-\nu$ (fixed atom), for each of the stationary points optimized for the 4-OT-catalyzed tautomerization of 2-oxo-4hexenedioate. (a) Plot of $\Delta \nu$ versus the normal mode index, with the modes ordered by increasing vibrational frequency, for each stationary point along the reaction pathway. (b) Plot of $\Delta \nu$ versus the frequency $\nu$ (fixed atom). 